\title{
Study of High Level Aminoglycoside Resistance among Enterococci in a Tertiary Care Centre, Navi Mumbai, India
}

\author{
Insha Irfan, S.A. Samant and Rakesh Kumar Mukhia*
}

Department of Microbiology, MGM Medical College and Hospital, Navi Mumbai, India

*Corresponding author

\author{
A B S T R A C T
}

\section{Keywords}

Enterococci, High level aminoglycoside resistance (HLAR).

Article Info

Accepted: 22 February 2017 Available Online: 10 March 2017
Enterococci, initially considered as normal commensal of intestinal tract have recently emerged as a medically important pathogen. Incidence of enterococccal infection is significantly high in patients suffering from urinary tract infection, blood stream infection and surgical sites infection. Nosocomial enterococcal infection is also common in organ transplants recipients cancer patients and debilitated patients receiving broad spectrum antibiotics. One of the important causes of development of multi drug resistant Enterococci is antibiotic selective pressure. This organism is considered as second leading cause of hospital acquired infections. The aim of the study was to know the high level aminoglycoside resistance among Enterococci in a tertiary care centre. The study was carried out over a period of 1 year. The isolated Enterococci were identified phenotypically followed by antibiotic susceptibility testing. Out of 2001 specimens showing bacterial growth, 50 strains of Enterococci (2.5\%) were isolated. Maximum $(3.22 \%)$ isolation of Enterococci was from urine samples. E. faecalis were $47(94 \%)$ and E. faecium were $3(6 \%)$. Out of 50 isolates of enteroccoci, 13 were resistant, 6 were intermediate and 31 were found sensitive. The isolates were found resistant or intermediate using high concentration Gentamicin disc were further tested for MIC level. It was found that 14 total isolates showed MIC $\geq 500 \mathrm{mcg}$. Enterococci show intrinsic low level cross resistance to all aminoglycosides due to decreased update of antibiotics. However recent report shows very high level of acquired resistence to even high level aminoglycoside. Hence all isolates of entrococci need to be tested for high level aminoglycosides.

\section{Introduction}

Enterococci are Gram-positive cocci (GPC) that often occur in pairs (diplococci) or short chains, and are difficult to distinguish from streptococci on physical characteristics alone. Two species are common commensal organisms in the intestines of humans: Enterococcus faecalis (90-95\%) and Enterococcus faecium (5-10\%) (Gilmore et $a l .$, 2002). Enterococci, initially considered as normal commensal of intestinal tract, have recently emerged as a medically important pathogen. Incidence of enterococccal infection is significantly high in patients suffering from urinary tract infection, blood stream infection and surgical sites infection. Nosocomial enterococcal infection is also common in organ transplants recipients cancer patients and debilitated patients receiving broad spectrum antibiotics (Sadar et al., 1994). One of the important causes of development of multi drug resistant Enterococci is antibiotic selective pressure. 
This organism is considered as second leading cause of hospital acquired infections (Yemisen et al., 2009). Enterococci show intrinsic resistance to cephalosporins, lincosamides, low levels of aminoglycosides, and many $\beta$-lactams, Enterococcus are also able to acquire resistance to many antibiotics by means of mutations or as a result of the transfer of genes located in plasmids/transposons or due to the incorporation of integrons. Infections by Enterococci have traditionally been treated with cell wall active agents in combination with an aminoglycoside. However resistance to low and high level aminoglycosides has been reported. Resistance to $\beta$ lactam antibiotics and vancomycin by some strains together with association of High Level Aminoglycoside Resistance (HLAR) with multi drug resistance (MDR) has lead to failure of synergistic effects of combination therapy (Patterson et al., 1990). The increasing role of Enterococcus in infections and their increasing resistance to antibiotics call for constant monitoring of their susceptibility (Sienko et al., 2014).

\section{Materials and Methods}

The study was carried out over a period of 1 year (October 2014 to September 2015) at the Department of Microbiology, MGM Medical College and Hospital, Kamothe, Navi Mumbai. Various Clinical specimens (Urine, Pus, Sputum, Blood, body fluids and miscellaneous samples including endotracheal tube, catheter tips) were taken from patients attending M.G.M. Medical College and hospital. Specimens were collected in a sterile, properly labelled container with aseptic precautions and processed as per the standard microbiological procedures.

Organism identification (Henry et al., 1998): All samples were screened for the presence of pus cells and organism.
Specimens were culture on Blood agar, MacConkey agar and incubated at $37^{\circ} \mathrm{C}$ for 24 hours. Growth was then processed for gram staining and catalase test. Gram positive cocci arranged in pairs showing catalase negative were considered as streptococcus species. Speciation of Enterococcus species was done by Gram's staining, Colony morphology, cultural characteristics of the colony and biochemical tests (Bile Esculin hydrolysis test, Pyrrolidonyl Arylamidase test, Resistance to Optochin and Bacitracin, Growth at $6.5 \% \mathrm{NaCl}$, Growth at $37^{\circ} \mathrm{C}$ and $45^{\circ} \mathrm{C}$, Hippurate hydrolysis test and Sugar Fermentation test). Gram stain of smear showed presence of Gram positive cocci, 1$1.5 \mu \mathrm{m} \times 0.5 \mu \mathrm{m}$, oval shaped arranged in pairs and short chains (Fig. 1).

\section{Antibiotic sensitivity testing}

Antibiotic Susceptibility testing was carried on the Brain Heart Infusion agar by KirbyBauer disc diffusion method. High level aminoglycoside resistance (HLAR) method was detected by following methods:

\section{Disc diffusion method (NCCLS, 2002)}

Colony of Enterococcus was inoculated into the Brain Heart Infusion (BHI) broth and incubated at $37^{\circ} \mathrm{C}$ for 4 hours. Growth was indicated by the appearance of turbidity in the medium. Turbidity of the medium was compared with 0.5 McFarland tube. Lawn culture was performed on BHI agar plate with the help of sterilized swab and Gentamicin disc $120 \mu \mathrm{g}$ was inoculated with a sterile forcep and incubated. A zone of $6 \mathrm{~mm}$ was considered resistant for Gentamicin.

\section{MIC Method}

Minimum inhibitory concentration of Gentamicin was determined by E-test. The strains which were resistant by disc diffusion 
method were checked by MIC. The colonies were inoculated in Brain Heart Infusion (BHI) broth. Growth was indicated by the appearance of turbidity which was compared with $0.5 \mathrm{McFarland}$ tube. Lawn culture was done on BHI agar plate with a sterile swab and E- strip was inoculated on BHI plate and incubated. All the results were interpreted according to CLSI guidelines. MIC $\geq 500 \mu \mathrm{g}$ for Gentamicin was considered as high level resistance.

\section{Results and Discussion}

In this study a total of 3144 clinical specimens were screened out of which 1143 $(36.4 \%)$ were sterile and 2001 (63.6\%) revealed bacterial growth. Out of 2001 (76.6\%) specimens showing bacterial growth, 50 strains of Enterococci (2.5\%) were isolated.

In our study maximum (3.22\%) isolation of Enterococci was from urine samples. It indicates that urinary tract infections are the most common infections caused by Enterococci in this set up. Out of 50 Enterococcus isolates, E. faecalis were 47 $(94 \%)$ and E. Faecium were $3(6 \%)$. E. faecalis is the most pathogenic species of Enterococci in our set up.

All the isolates of Enterococci were subjected to test for High Level Aminoglycoside Resistance (HLAR) by two methods namely Gentamicin high concentration disc diffusion method and MIC method.

The results of Gentamicin resistance as observed by high concentration Gentamicin discs $(120 \mu \mathrm{g})$ has been shown in table 1. It was observed that total 14 isolates were resistant to Gentamicin as their MIC was $\geq$ $500 \mu \mathrm{g}$. One isolate which showed intermediate sensitivity by High Concentration Gentamicin Disc exhibited $\mathrm{MIC}>500 \mu \mathrm{g}$.

Table.1 Gentamicin resistance observed in Enterococci by high concentration $(120 \mathrm{mcg})$ gentamicin

\begin{tabular}{|c|c|c|c|c|}
\hline Isolates & Total & Resistant & Sensitive & Intermediate \\
\hline E. faecalis & 47 & $13(27.6 \%)$ & $31(65.95 \%)$ & $3(6.38 \%)$ \\
\hline E. faecium & 03 & $01(33.3 \%)$ & $0(0 \%)$ & $02(66.7 \%)$ \\
\hline TOTAL & 50 & $14(28 \%)$ & $31(62 \%)$ & $5(10 \%)$ \\
\hline
\end{tabular}

Table.2 HLAR (High Level Aminoglycoside Resistance) detection by MIC method

\begin{tabular}{|c|c|c|c|}
\hline Total isolates & $\begin{array}{l}\text { Sensitive by Gentamicin } \\
\text { disc diffusion method } \\
(\mathbf{1 2 0} \boldsymbol{\mu g})\end{array}$ & $\begin{array}{l}\text { Intermediate by } \\
\text { Gentamicin Disc } \\
(\mathbf{1 2 0} \boldsymbol{\mu g})\end{array}$ & HLAR (MIC $\geq \mathbf{5 0 0} \boldsymbol{\mu g})$ \\
\hline 50 & $31(62 \%)$ & $5(10 \%)$ & $14(28 \%)$ \\
\hline
\end{tabular}


Fig.1 Positive and negative BEA

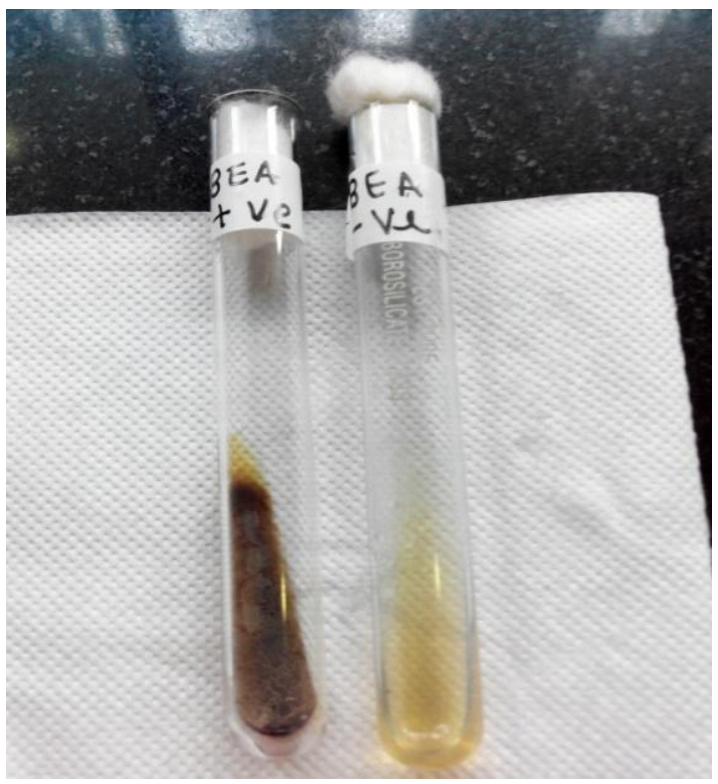

Fig.3 Enterococcus with $\mathrm{MIC} \geq 500 \mu \mathrm{g}$

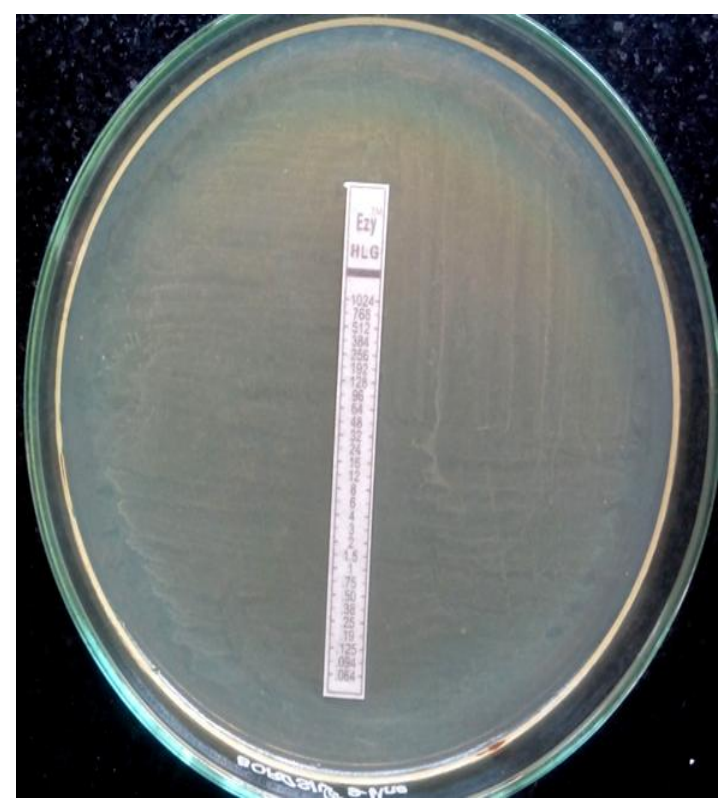

Fig.2 Antibiotic sensitivity of Gentamicin disc $(120 \mu \mathrm{g})$

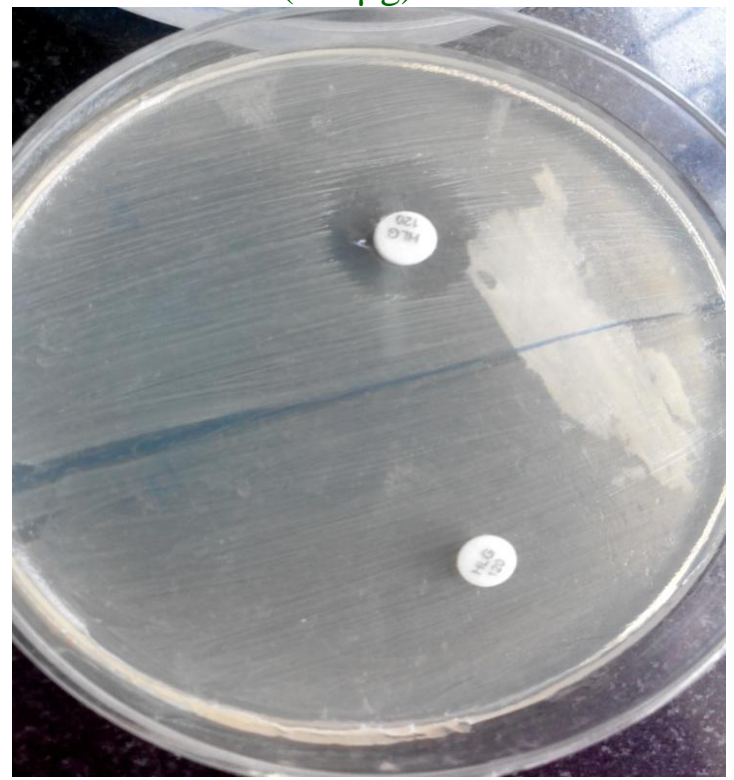

Fig.4 Enterococcus with $\mathrm{MIC} \leq 500 \mu \mathrm{g}$

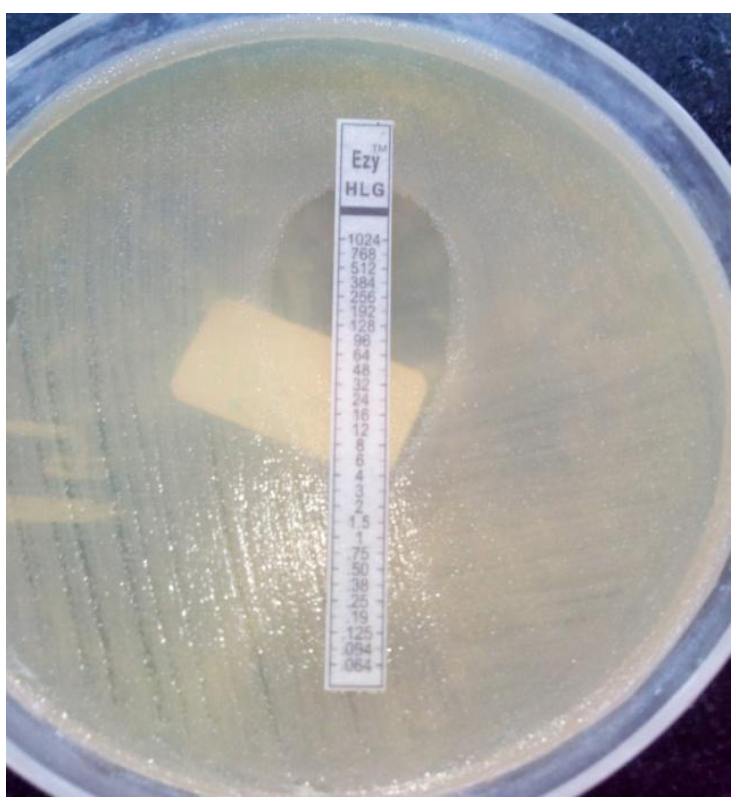


Fig.5 Rate of isolation of Enterococci from various specimens

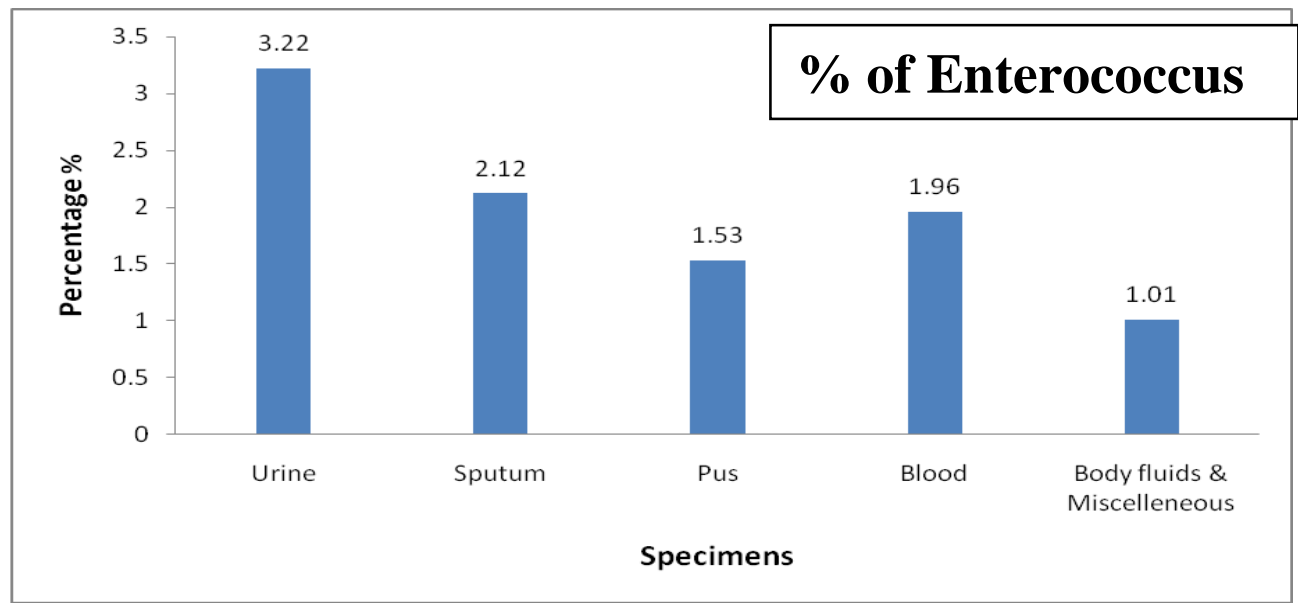

Fig.6 Antibiotic sensitivity pattern of Enterococcus faecalis

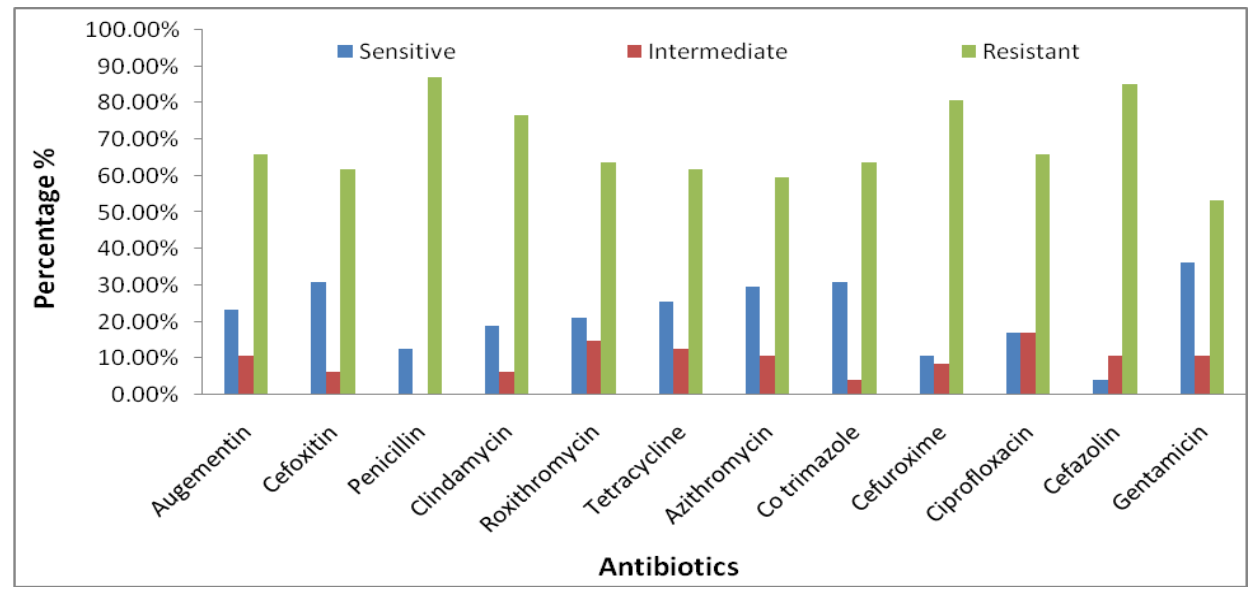

Fig.7 Antibiotic sensitivity pattern of Enterococcus faecuim

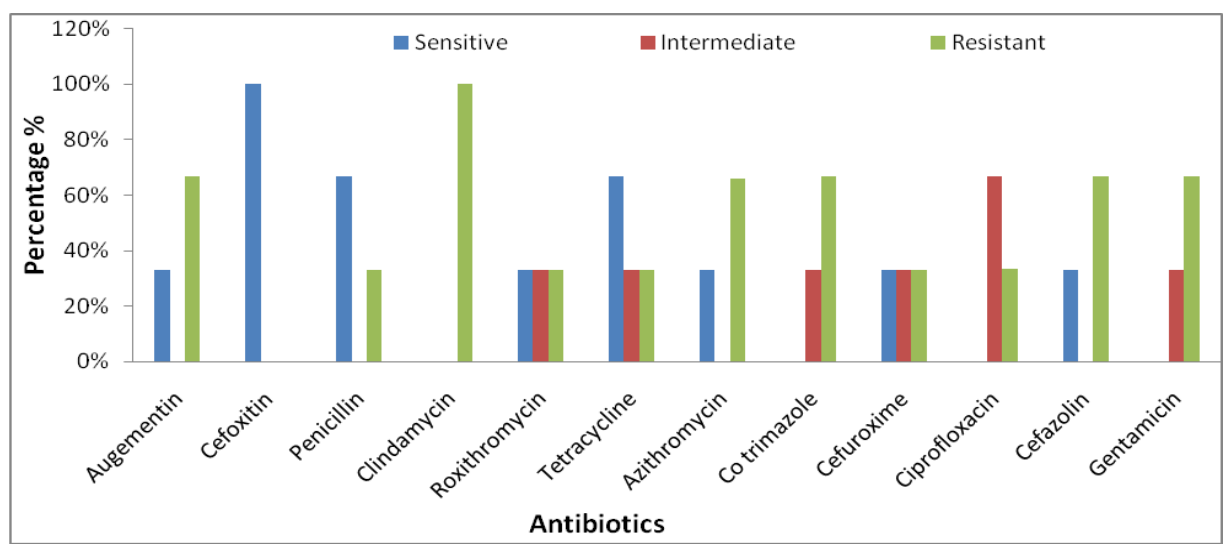


Recent years have witnessed increased interest in Enterococci not only because of their ability to cause serious infections but also because of their increasing resistance to many antimicrobial agents. Glycopeptideresistant Enterococci have become a major threat to hospitalized patients. In this study a total of 3144 clinical specimens were screened out of which $1143(36.4 \%)$ were sterile and $2001(63.6 \%)$ revealed bacterial growth. The highest bacterial growth $61.5 \%$ was isolated from urine samples, followed by sputum samples with bacterial growth of $97 \%$. Bacterial growth was also observed from $83.5 \%$ of pus samples, $82.5 \%$ of body fluids and miscellaneous samples and $10.78 \%$ of blood samples. Enterococcus were isolated from $3.22 \%$ of urine samples, $2.12 \%$ of sputum samples, $1.96 \%$ of blood samples, 1.53 of pus samples and $1.01 \%$ of body fluids and miscellaneous specimens (Endotracheal tip, catheter tip etc). This shows that maximum $(3.22 \%)$ isolation of Enterocooci was isolated from urine samples. It indicates that urinary tract infections are the most common infections caused by Enterocooci in this set up. Seema et al., (2008) showed that maximum number of Enterococci were isolated from urine samples $62.36 \%$ followed by $27.02 \%$ from blood, $1.83 \%$ from pus and $0.36 \%$ from body fluids (Fig. 5).

Amongst Enterococcus from urine samples Enterococcus faecalis was $93.75 \%$ and Enterococcus faecuim was $6.25 \%$. Among sputum samples there was isolation of only one species of Enterococcus i.e. Enterococcus faecalis. Amongest the Enterococci isolated from pus samples, $66.6 \%$ were E. faecalis and $33.4 \%$ were E. faecuim.

In specimens like blood, body fluids and miscellaneous samples only E. faecalis was isolated. This shows that Enterococcus faecalis is the most pathogenic species of Enterococci in our set up. Bose et al., (2012) showed that during the study period of one and half year, 544 Enterococcus species were isolated among which $446(82 \%)$ were $E$. faecalis and 98 (18\%) were E. faecuim. Shouten et al., (2014) also found $83 \%$ E. faecalis and $13,6 \%$ E. faecuim isolates in their study. This indicates that $80-90 \%$ of all enterococcal infections were caused by $E$. faecalis.

Antibiotic sensitivity of E. faecalis which shows that $23.4 \%$ isolates of E. faecalis were sensitive to Augmentin and $65.9 \%$ were resistant. $17 \%$ isolates of E. faecalis were sensitive to Ciprofloxacin and $65.9 \%$ were resistant. $12.7 \%$ of isolates of E. faecalis were seen sensitive to Penicillin and $87.2 \%$ were resistant.36.17\% isolates of E. faecalis were sensitive to Gentamicin and $53.9 \%$ were resistant (Fig. 2). Hasani et al., (2012) showed that $96.3 \%$ strains were resistant to Ampicillin, $61.1 \%$ strains were resistant to Vancomycin. 93.5\% strains were resistant to Ciprofloxacin and $98.1 \%$ strains were resistant to Penicillin.

Latika Shah et al., (2012) showed the antimicrobial susceptibility pattern which shows that $56 \%$ isolates of E. faecuim were sensitive to Penicillin and $44 \%$ were resistant. $60 \%$ isolates of E. faecuim were sensitive to Ampicillin and $40 \%$ were resistant. $47 \%$ isolates of E. faecuim were sensitive to Gentamicin and $53 \%$ were resistant. $38 \%$ of isolates of E. faecium were sensitive to Ciprofloxacin and 38\% were resistant (Figs. 6 and 7).

Our study shows that E. faecalis is more resistant to routine antibiotics as compared to E. faecuim. However many studies have also demonstrated that E. faecuim is more resistant than E. faecalis.

Gentamicin Resistance observed in Enterococci by high concentration disc (120mcg) which shows that out of 47 strains, $65.95 \%$ isolates of $E$. faecalis were sensitive 
to Gentamicin, $6.38 \%$ showed intermediate zone and $27 \%$ isolates were resistant. In $E$. faecuim out of 3 strains 1 isolate of $E$. faecuim were seen sensitive to Gentamicin and 2 were resistant. Sienko et al., showed a total of 85 isolates of Enterococcus in which 47 were Enterococcus faecalis and 38 were Enterococcus faecalis. E. faecalis showed susceptibility to all aminoglycosides, whereas E. faecuim strains were not susceptible to Gentamicin.

MIC level of Gentamicin against all 19 isolates of Enterococci which were resistant or intermediately sensitive to high content disc of Gentamicin $(120 \mu \mathrm{g})$, it was observed that only 14 isolates showed resistance to High Level Aminoglycoside Resistance as their MIC was $\geq 500 \mu \mathrm{g}$. One isolate which showed intermediate sensitivity to high content Gentamicin $(120 \mu \mathrm{g})$ also showed MIC $\geq 500 \mu \mathrm{g}$. Sienko et al., showed that high level resistance to aminoglycosides resistance to Gentamcin was detected in $36 \%$ of $E$. faecalis. E. faecalis strains showed susceptibility to all aminoglycosides, whereas E. faecuim strains were not susceptible to both Gentamicin and Streptomycin (Figs. 2 and 3 ).

Comparison of high content disc method and MIC method for detection of HLAR (High Level Aminoglycoside Resistance), it was found that out of 19 isolates which were resistant to high content disc of Gentamicin $(120 \mu \mathrm{g})$ only 14 isolates had MIC $\geq$ $500 \mu \mathrm{g} / \mathrm{ml}$. It has been reported that agar screen method must be used to confirm HLAR in Enterococci (Adhikari et al., 2010). MIC method is analogous to agar screen method. Also it was found more specific and superior to disc diffusion method (Table 2).

In conclusion Enterococci show intrinsic low level cross resistance to all aminoglycosides due to decreased update of antibiotics. However the recent report shows very high level of acquired resistence to even high level aminoglycoside. Hence all isolates of entrococci need to be tested for high level aminoglycosides.

Out of 50 isolates of enteroccoci, 13 were resistant, 6 were intermediate and 31 were found sensitive. The isolates were found resistant or intermediate using high concentration Gentamicin disc were further tested for MIC level. It was found that 14 total isolates showed MIC $\geq 500 \mathrm{mcg}$. This is because disc diffusion method may not detect borderline resistance (Fig. 4).

The clinical laboratories usually use disc diffusion test for antimicrobial susceptibility testing. However, disc diffusion technique may fail to detect actual resistance. Hence it is necessary to test MIC of Gentamicin.

Drug resistant Enterococci present a challenge to the clinicians and clinical microbiologist because of the increase occurrence in nosocomial infections. This obligates the clinical microbiologist to detect inherent antibiotic resistance and identify the most useful active antibiotic treatment.

The present study highlighted the importance of high occurrence of HLAR in this set up which necessitates its routine testing. Alternative regimes in the management of enterococcal infection need to be evaluated.

\section{Ethical clearance}

The study was cleared by institutional ethics committee of MGM Institute of Health Sciences, Navi Mumbai and written consent from the patients was taken prior to collection of samples.

\section{Acknowledgement}

We want to thank the Dean and Medical Superintendent (Mahatma Gandhi Mission's 
Medical College and Hospital, Navi Mumbai) for their support and permission. Also thanks to my HOD Dr. A.D. Urhekar and all staff of Microbiology Department, MGM Medical College, Navi Mumbai for their valuable suggestion and support.

\section{References}

Adhikari, L. 2010. HLAR and reduced susceptibility to Vancomycin in nosocomial enterococci. J. Global Infect. Dis., 2(3): 231-35.

Bose, S., Ghosh, K., Barapatre, R. 2012. Prevalance of Drug Resistance Among Enterococcus spp Isolated From A Tertiary Care Hospital, (1): 44.

Centinkaya, Y., Falk, P., Mayhall, C.G. 2000. Vancomycin resistant Enterococci. Clin. Microbiol. Rev., 13: 686-707.

Chen, A.Y., Zerros, J.M. 2009. Antimicrobial Drug Resistance and Infectious Disease: Humana Press; Chapter 49, Enterococcus: Anti-microbial Resistance in Enterococci. Epidemiology, Treatment and Control, pp. 715-33.

Ghoshal, U., Garg, A., Tiwari, D.P., Ayyagari, A. 2006. Emerging vancomycin resistance in Enterococci in india. Indian J. Pathol. Microbiol., 49: 620-2.

Gilmore, M.S., Coburn, P.S., Nallapareddy, S.R., et al. 2002. The Enterococci: Pathogenesis, Molecular Biology, and Antibiotic Resistance. Washington, D.C.: $\quad$ ASM Press, ISBN 9781555812348.

Hasani, A., Y. Sharifi, R. Ghotaslou, B. Naghili, M. Aghazadeh, M. Milani, A. Bazmani. 2012. Molecular screening of virulence genes in high level gentamicin resistant Enterococcus faecalis and Enterococcus faecuim isolated from clinical specimens in Northwest Iran, 30(2): 175-81.
Henry, D. 1998. Isenberg. Essential Procedures for Clinical Microbiology. Collection, Transport and Manipulation of Clinical Specimens. ASM Press Washington, D.C. Page no. 3-35.

NCCLS. 2002. Performance Standards for antimicrobial susceptibility testing; Twelfth Informational Supplement. NCCLS document M100-S12 [ISBN 156238-454-]. NCCLS, 940 West Valley Road, Suite 1400, Wayne, Pennsylvania 19087-1898 USA.

Patterson, J.E., Zervos, M. High level gentamicin resistance in Enterococcus: microbiology, genetic basis and epidemiology. Rev. Infect. Dis., 12:6 44-51.

Sadar, H.S., Pfaller, M.A., Tenover, F.C., Hollis, R.J., Jones, R.N. 1994. Evaluation and characterization of multiresistant Enterococcus faecium from 12 US medical centers. J. Clin. Microbiol., 32: 2840 -2.

Seema Sood, Meenakshi Malhotra, B.K. Das and Arti Kapil. 2008. Enterococcal infections and antimicrobial resistance. Indian J. Med. Res., 128: 111-121.

Sekar, R., Srivani, R., Vignesh, R., Kownhar, H., Shankar, E.M. 2008. Low recovery rates of high level aminoglycoside resistant Enterococci could be attributable to restricted usage of aminoglycosides. J. Med. Microbiol., 57: 397-8.

Shah, L., Mulla, S., Patel, K., Rewadiwala, S. 2012. Prevalence of Enterococci With Higher Resistance Level in a Teritiary Care Hospital, 2249-4995.

Shouten, M.A., M. Milani, D. Ojdana. 2014. High level aminoglycoside resistant Enterococcus, Vol 6(03): 16-18.

Sienko, A., Wieczorek, A., Sacha, P., Majewski, P., Ojdana, D., Tryniszewska, E. 2014. Occurrence of high level aminoglycoside resistance 
among Enterococcus species strains Vol 4(1): 179-187.

Udo, E.E., AL-Sweih, N., Philips, O.A., Chugh, T.D. 2003. Species prevalence and antibacterial resistance of Enterococci isolated in Kuwait hospitals. J. Med. Microbiol., 52: 16338.
Yemisen, M., Demirel, A., Mete, B., Kaygusuz, A., Mert, A., Tabak, F., Ozturk, R. 2009. Comparative in vitro antimicrobial activity of tigecycline against clinical isolates of vancomycin resistant Enterococcus. Indian J. Med. Microbiol., 27: 373- 83.

\section{How to cite this article:}

Insha Irfan, S.A. Samant and Rakesh Kumar Mukhia. 2017. Study of High Level Aminoglycoside Resistance among Enterococci in a Tertiary Care Centre, Navi Mumbai. Int.J.Curr.Microbiol.App.Sci. 6(3): 1612-1620. doi: https://doi.org/10.20546/ijcmas.2017.603.186 